\title{
ASYMPTOTIC VALUES OF SUBHARMONIC FUNCTIONS
}

\section{J. L. FERNÁNDEZ, J. HEINONEN, and J. G. LLORENTE}

[Received 19 January 1995-Revised 19 September 1995]

\section{Introduction}

In this paper we study the boundary behaviour of subharmonic functions in $\mathbb{B}^{n}$, the unit ball of $\mathbb{R}^{n}$, or in the upper half space of $\mathbb{R}^{n+1}, \mathbb{R}_{+}^{n+1}=\left\{(x, y): x \in \mathbb{R}^{n}\right.$, $y>0\}$.

A function $u$ defined in $\mathbb{B}^{n}$ (respectively, $\mathbb{R}_{+}^{n+1}$ ) has asymptotic value $\lambda \in$ $[-\infty, \infty]$ at the point $x \in \partial \mathbb{B}^{n}$ (respectively, $\mathbb{R}^{n}$ ) if there is a path $\gamma(t), 0 \leqslant t<1$ contained in $\mathbb{B}^{n}$ (respectively, $\mathbb{R}_{+}^{n+1}$ ) and ending at $x$ such that $u(p)$ tends to $\lambda$ as long as $p$ tends to $x$ along $\gamma$, that is, $\gamma(t) \rightarrow x$ as $t \rightarrow 1$ and $u(\gamma(t)) \rightarrow \lambda$ as $t \rightarrow 1$.

The set of $x \in \partial \mathbb{B}^{n}$ (respectively, $\mathbb{R}^{n}$ ) at which $u$ has an asymptotic limit is denoted by $A(u)$. We will be particularly interested in the case $\lambda=\infty$, and the corresponding subset of $\partial \mathbb{B}^{n}$ (respectively, $\mathbb{R}^{n}$ ), consisting of the points at which $u$ has asymptotic value $+\infty$, will be denoted by $A(u,+\infty)$.

If $\gamma$ is restricted to be a radius (respectively, a vertical line) and $u$ has a finite radial (vertical) limit at $x$ then $x \in \partial \mathbb{B}^{n}$ (respectively, $\mathbb{B}^{n}$ ) is said to be a Fatou point of $u$. We write $F(u)$ to denote the set of all Fatou points of $u$.

These definitions have their origin in the case of the unit disk

$$
\Delta=\{z \in \mathbb{C}:|z|<1\} .
$$

For an analytic function $f$ in the unit disk, $F(f)$ (respectively, $A(f, \infty)$ ) denotes the set of points of the unit circle at which $f$ has finite radial limit (respectively, $|f|$ has asymptotic value $\infty$ ). If $u$ is subharmonic in $\Delta$, then the above definitions of radial and asymptotic limits apply,

G. R. McLane [18] realized that the boundary behaviour of some classes of analytic functions in the unit disk was more easily understood by considering not only radial limits but also asymptotic limits. He introduced the class of analytic functions in $\Delta$ having asymptotic values at a dense set of the unit circle, now called the McLane class. The analogous class for subharmonic functions was studied by Hornblower [12] and he gave a sufficient growth condition for a subharmonic function to be in this class. More precisely, a subharmonic function $u$ in $\Delta$ is in the McLane class if

$$
\int_{0}^{1} \log ^{+} M(r) d r<\infty
$$

where $M(r)=\sup \{u(z):|z|=r\}$. (See $[13,11]$.) Barth, Rippon and Sons $[4]$ have

Research of the first and third authors supported by a grant from CICYT, Ministerio de Educación y Ciencia, Spain, and a grant from the EU HCM contract number CHRX-CT92-0071.

Research of the second author supported by a grant from National Science Foundation, U.S.A. and the Academy of Finland.

1991 Mathematics Subject Classification: 31B25.

Proc. London Math. Soc. (3) 73 (1996) 404-430. 
improved the conclusions of Hornblower's theorem in the analytic case and obtained the following theorem.

THEOREM BRS. If $f$ is analytic in the unit disk and satisfies Hornblower's condition

$$
\int_{0}^{1} \log ^{+} \log ^{+} M(r, f) d r<\infty,
$$

where $M(r, f)=\sup \{|f(z)|:|z|=r\}$, then for any arc I of the unit circle, either

$$
|F(u) \cap I|>0 \text { or } A(u,+\infty) \cap I \neq \varnothing .
$$

Here and hereafter $|\cdot|$ always means Lebesgue measure in the corresponding euclidean space.

In higher dimensions we have the following extension.

THEOREM 1. Let u be subharmonic in $\mathbb{B}^{n}$, satisfying Hornblower's condition

$$
\int_{0}^{1} \log ^{+} M(r) d r<\infty
$$

where $M(r)=\sup \{u(y):|y| \leqslant r\}$. Then, for every ball $B$ in $\partial \mathbb{B}^{n}$, either

$$
|F(u) \cap B|>0 \text { or } A(u,+\infty) \cap B \neq \varnothing .
$$

As a matter of fact, we will prove the following version of Theorem 1 in the upper half space of $\mathbb{R}_{+}^{n+1}$.

THEOREM 1'. Let $u$ be subharmonic in $\mathbb{R}_{+}^{n+1}$. Let

$$
M(t)=\sup \{u(x, y):|x| \leqslant 1, t \leqslant y \leqslant 1\}
$$

and suppose that $u$ satisfies Hornblower's condition

$$
\int_{0}^{1} \log ^{+} M(t) d t<\infty .
$$

Then, for every ball $B$ in $\mathbb{R}^{n}$, which is contained in $\mathbb{B}^{n}$, either

$$
|F(u) \cap B|>0 \text { or } A(u,+\infty) \cap B \neq \varnothing .
$$

It is convenient to point out that these results in the ball are not deduced from the corresponding ones in the upper half space, or vice versa. However, the proofs are completely parallel and, since technical details are perhaps simpler in $\mathbb{R}_{+}^{n+1}$, we have preferred to deal with Theorem $1^{\prime}$ instead of Theorem 1 .

The sharpness of condition $(H)$ is discussed in [11, Example 10.15], in the context of the unit disk. To wit, for every positive increasing function $k(r)$, with $0 \leqslant r<1$, such that

$$
\limsup _{r \rightarrow 1}(1-r) \log k(r)>0 \text {, }
$$


a subharmonic function $u$ in $\Delta$ is constructed so that its maximum $M(r)$ satisfies $M(r)<k(r)$ and $u$ is not in the McLane class. Note that condition $(\mathrm{H})$ forces $(1-r) \log ^{+} M(r) \rightarrow 0$. These examples can be easily extended to higher dimensions by adding dummy variables.

As a consequence of Theorems 1 and $1^{\prime}$, we have the following extensions of Hornblower's theorems in $\mathbb{B}^{n}$ and $\mathbb{R}_{+}^{n+1}$.

COROllaRy 1. If $u$ is subharmonic in $\mathbb{B}^{n}$ (respectively, $\mathbb{R}_{+}^{n+1}$ ) and $u$ satisfies condition $(\mathrm{H})$ (respectively, $\left(\mathrm{H}^{\prime}\right)$ ) then $u$ has asymptotic limits at a dense subset of $\partial \mathbb{B}^{n}$ (respectively, $\mathbb{R}^{n}$ ).

There are different approaches to the proof of Hornblower's theorem in the unit disk. They are usually divided into a topological part, namely a characterization of the McLane class in terms of Koebe arcs (see [11]), and a harmonic measure argument. Such a clear division is not possible in higher dimensions; for instance, the topological part fails entirely. What we have done is to combine both parts in a unified, less direct, argument (see Lemma 6). The harmonic measure argument that we use is inspired by a proof of Hornblower's theorem due to P. Rippon [11, p. 798] which relies on a harmonic measure estimate in a special domain. However, it is perhaps worth mentioning that some techniques usually employed to estimate harmonic measure in the plane (conformal mapping, Ahlfors distortion theorem, etc.) are no longer available in higher dimensions.

An analytic function $f$ in the unit disk is said to be a Bloch function if

$$
\sup \left\{(1-|z|)\left|f^{\prime}(z)\right|:|z|<1\right\}<\infty .
$$

Results of Anderson and Pitt [1] and Makarov [17] show that if a Bloch analytic function in the unit disk has a small Fatou set (of measure zero) then it must have infinite radial limits at a relatively large set (of Hausdorff dimension 1). Some results of Berman [5] and Fernández and Llorente [8] have extended that fact to functions with less restrictive growth, the conclusions involving asymptotic limits instead of radial limits. Barth and Rippon [3] have recently obtained a common improvement of these last two results from which the following theorem, that we state below as Theorem BR, is deduced. Recall that if $E$ is a subset of $\mathbb{R}^{n}$ and $\beta \geqslant 0$ then the Hausdorff $\beta$-content of $E, M_{\beta}(E)$, is defined as

$$
\inf \left\{\sum_{j} r_{j}^{\beta}\right\}
$$

where the infimum is taken over all coverings of $E$ by balls $\left\{B_{j}\right\}$ of radius $\left\{r_{j}\right\}$. The Hausdorff dimension of $E$ can be defined by

$$
\operatorname{dim} E=\inf \left\{\beta \geqslant 0: M_{\beta}(E)=0\right\} .
$$

Note that $M_{\beta}(E)>0$ implies $\operatorname{dim} E \geqslant \beta$.

THEOREM BR. Suppose that $f$ is analytic in $\Delta$, with

$$
u(z)=\log |f(z)| \leqslant \frac{C}{(1-|z|)^{\alpha}}
$$


for some positive constant $C>0$ and some $\alpha \in[0,1]$. Then, if $I$ is any arc of the unit circle, either

$$
|F(f) \cap I|>0 \text { or } \quad M_{1-\alpha}(A(u,+\infty) \cap I) \geqslant|I|^{1-\alpha} .
$$

If this last possibility occurs, then, in particular, $\operatorname{dim}(A(u,+\infty) \cap I) \geqslant 1-\alpha$.

Barth and Rippon have used fine potential theory to prove some analogous results for subharmonic functions in $\Delta$. The next theorem, that we state only in $\mathbb{R}_{+}^{n+1}$, extends the result of Barth and Rippon to higher dimensions.

Hereafter, for $\alpha \geqslant 0$, we will denote by $\mathcal{M}_{\alpha}$ the class of subharmonic functions in $\mathbb{R}_{+}^{n+1}$ which, for some constant $C_{u}$, satisfy a growth condition of the type:

$$
u(x, y) \leqslant C_{u} / y^{\alpha}, \text { for each } x \in \mathbb{R}^{n} \text {, and each } y \text { such that } 0<y<1 \text {. }
$$

Theorem 2. Suppose that $u \in \mathcal{M}_{\alpha}$ for some $\alpha \in[0,1]$. If $B$ is a ball in $\mathbb{R}^{n}$, then either

$$
|F(u) \cap B|>0
$$

or

$$
M_{n-\alpha}(A(u,+\infty) \cap B) \geqslant M_{n-\alpha}(B) .
$$

If this last possibility occurs, then, in particular,

$$
\operatorname{dim}(A(u,+\infty) \cap B) \geqslant n-\alpha .
$$

It is often useful to interpret problems on boundary behaviour in terms of martingales. If $p \in \mathbb{N}$, a $p$-adic martingale (respectively, submartingale) in the unit cube $Q_{0}=[0,1]^{n}$ is a sequence $\left(S_{k}\right)$ of step functions in $Q_{0}$, each $S_{k}$ being constant on the $k$-generation cubes of the form

$$
\prod_{j=1}^{n}\left[m_{j} p^{-k},\left(m_{j}+1\right) p^{-k}\right) \quad \text { where } 0 \leqslant m_{j} \leqslant p^{k}-1,
$$

and such that the value of $S_{k}$ at every cube $Q_{k}$ of the $k$-generation is equal (respectively, less than or equal) to the mean of the values which $S_{k+1}$ takes at the $p^{n}$ subcubes of the $(k+1)$-generation in which $Q_{k}$ is divided.

Because of the metric nature of the problems in which we are interested, we will restrict our attention to these special cases of submartingales. For further general information about martingales and their abstract definition, we refer to $[20,22]$.

For a submartingale $\mathscr{S}=\left(S_{k}\right)$ in the unit cube $Q_{0}$ we define, following the analogy with the function-theoretical case, the Fatou set $F(\mathscr{S})$ and the divergence set $A(\mathscr{S},+\infty)$ as

$$
\begin{aligned}
F(\mathscr{S}) & =\left\{x \in Q_{0}: \lim _{k} S_{k}(x) \text { exists and is finite }\right\}, \\
A(\mathscr{S},+\infty) & =\left\{x \in Q_{0}: \lim _{k} S_{k}(x)=+\infty\right\} .
\end{aligned}
$$

The next result, due to Makarov [17], is a discrete analogue of the Bloch continuous case. 
THEOREM C. Let $\left(S_{k}\right)$ be a dyadic martingale in $[0,1]$ such that its increments, $S_{k}-S_{k-1}$, are uniformly bounded. If I is any subinterval of $[0,1]$ then, either

$$
|F(\mathscr{S}) \cap I|>0 \text { or } \operatorname{dim}(A(\mathscr{S},+\infty) \cap I)=1 .
$$

In view of this result, it is natural to ask if there exists some kind of analogue of Theorem 2 for submartingales. The answer is given in the following theorem, which extends Theorem C.

THEOREM 3. Let $\mathscr{S}=\left(S_{k}\right)$ be a p-adic submartingale in the unit cube $Q_{0}$ of $\mathbb{R}^{n}$ such that the growth condition

$$
S_{k} \leqslant C p^{k \alpha}
$$

holds for some $\alpha \geqslant 0$, and $C>0$, uniformly in $k$. If $Q$ is a cube contained in $Q_{0}$, then either

$$
|F(\mathscr{S}) \cap Q|>0
$$

or

$$
M_{n-\alpha}(A(\mathscr{Y},+\infty) \cap Q) \geqslant K M_{n-\alpha}(Q)
$$

for some positive constant $K$, depending only on $n$ and $p$. If this last possibility occurs, then, in particular,

$$
\operatorname{dim}(A(\mathscr{S},+\infty) \cap Q) \geqslant n-\alpha .
$$

Concerning the sharpness of Theorem 2, we give the following partial answer.

THEOREM 4. For each $\alpha$, with $0<\alpha<1$, there exists a harmonic function $u$ in $\mathcal{M}_{\alpha}$ such that $|F(u)|=0$ and that, for any ball $B$ in $\mathbb{R}^{n}$, we have

$$
\begin{array}{cl}
\operatorname{dim}(A(u,+\infty) \cap B)=n-\alpha & \text { if } \alpha \leqslant \frac{1}{2}, \\
n-\alpha \leqslant \operatorname{dim}(A(u,+\infty) \cap B) \leqslant n-2 \alpha(1+2 \alpha)^{-1} & \text { if } \alpha>\frac{1}{2} .
\end{array}
$$

The proof of Theorem 4 reduces to the case $n=1$ (see Theorem $4^{\prime}$ in $\S 5$ ). A martingale approach has proved to be very useful in constructing these examples. Indeed, the procedure that we have followed is to construct a martingale, sharp in Theorem 3 (for $n=1$ ), and then to translate it into a harmonic function in the upper half plane. The proof combines some techniques appearing in [15] in the case of radial limits with an argument of Berman [5, Theorem 2] which allows one to replace radial limits by asymptotic limits. It turns out that the barrier at the value $\frac{1}{2}$ is not present in the martingale situation and is, therefore, a consequence of the transfer method. We refer to $\S 5$ for further details.

The paper is organized as follows: $\S 1$ contains some preliminary lemmas; in $\S 2$ we give some harmonic measure estimates needed in the proofs of Theorems 1' and 2 , which are proved in $\S 3$, under the additional assumption that the functions involved are continuous. Theorem 3 is proved in $\S 4$, and finally, Theorem 4 is proved in $\S 5$. In $\S 6$ we describe the changes needed in the proofs of Theorems $1^{\prime}$ and 2 to cover the discontinuous case.

We would like to thank D. Burkholder and R. Berman for some valuable correspondence. We also thank P. Rippon for helpful conversations and for 
sending us the preprint [3]. Part of this research was done when the second author was visiting Universidad Autónoma de Madrid in December 1992. He wants to thank its Mathematics Department for its hospitality.

Recently, following the distribution of the present paper in preprint form, two interesting results have been obtained which extend and complement ours: S. J. Gardiner ('Asymptotic values and minimal fine limits of subharmonic functions of slow growth') has extended Theorem 2 to the full range $[0, n]$ of $\alpha$, while, on the other hand, J. M. Wu ('Growth and asymptotic sets of subharmonic functions') has also obtained a partial extension of the range of $\alpha$ in Theorem 4 .

\section{Preliminary lemmas}

Let us fix some notation which we shall use throughout this paper.

Points in $\mathbb{R}_{+}^{n+1}$ are denoted by $(x, y)$, with $x \in \mathbb{R}^{n}$ and $y \in \mathbb{R}^{+}$.

For $a \in \mathbb{R}^{n}$ and $r>0, B^{n}(a, r)$ denotes the open euclidean ball in $\mathbb{R}^{n}$ of centre $a$ and radius $r$. We shall denote $B^{n}(0,1)$ by $\mathbb{B}^{n}$.

The boundary of a set $E$ is denoted by $\partial E$. Associated to a ball $B^{n}(a, r)$ in $\mathbb{R}^{n}$ we define the Carleson cylinder $C(a, r)$ as $B^{n}(a, r) \times(0, r)$ and the tent $T(a, r)$, which consists of the points $(x, y)$ with $x \in B^{n}(a, r)$ and $0<y<\operatorname{dist}\left(x, \partial B^{n}(a, r)\right)$. In general, if $a \in \mathbb{R}^{n}$ and $r, h$ are positive numbers, then $C(a, r, h)$ will be the cylinder $B^{n}(a, r) \times(0, h)$.

Given a function $f$ (respectively, a measure $\mu$ ) on $\mathbb{R}^{n}$ then $P[f]$ (respectively, $P[\mu]$ ) is the Poisson extension of $f$ (respectively, $\mu$ ) to $\mathbb{R}_{+}^{n+1}$, that is,

$$
P[f](x, y)=\int_{\mathbb{R}^{n}} P(x-t, y) f(t) d t
$$

where $P(x, y)=K_{n} y\left(|x|^{2}+y^{2}\right)^{-\frac{1}{2}(n+1)}$ is the Poisson kernel in $\mathbb{R}_{+}^{n+1}\left(K_{n}\right.$ is a normalizing constant), and analogously

$$
P[\mu](x, y)=\int_{\mathbb{R}^{n}} P(x-t, y) d \mu(t) .
$$

Finally, if $u$ is a function in $\mathbb{R}_{+}^{n+1}$ and $x \in \mathbb{R}^{n}$ is a Fatou point of $u$, we put $u^{*}(x)=\lim _{y \rightarrow 0} u(x, y)$.

The next lemma is an extension due to Dahlberg of a classical theorem of Littlewood.

Lemma D (Dahlberg [7]). Let $\Omega \subset \mathbb{R}^{n+1}$ be the domain above the graph of a Lipschitz function in $\mathbb{R}^{n}$, and let $u$ be a subharmonic function in $\Omega$. If $u$ is bounded above in $\Omega$, then $u$ has finite vertical limits at almost all points of $\partial \Omega$ with respect to the surface measure in $\partial \Omega$.

Lemma 1. Let $\beta \in[0, n]$ and $E \subset \mathbb{B}^{n}$ with $M_{\beta}(E)<1$. Then there exists $a$ countable collection of balls, $\left\{B_{k}=B^{n}\left(a_{k}, r_{k}\right)\right\}$ in $\mathbb{R}^{n}$, such that the following conditions hold:

(i) $E \subset \cup_{k} B_{k}$; 
(ii) $\sum_{k} r_{k}^{\beta}<1$;

(iii) $\left|\mathbb{B}^{n}-\cup_{k} B_{k}\right|>0$.

Proof. Assertions (i), (ii) are immediate from the definition of the $\beta$-content. Condition (iii) follows since $\sum r_{k}^{\beta}<1$ implies $\sum r_{k}^{n}<1$.

Lemma 2 (Berman [5]). Let $\left\{B_{k}=B^{n}\left(a_{k}, r_{k}\right)\right\}$ be a covering of a set $E \subset \mathbb{B}^{n}$ such that, for some $\beta \in[n-1, n]$,

$$
\sum_{k} r_{k}^{\beta}<\infty
$$

Then there exists a function $b$, harmonic and positive in $\mathbb{R}_{+}^{n+1}$, with

$$
b(x, y) \geqslant 1 / y^{n-\beta}
$$

for every $(x, y) \in \bigcup_{k} S_{k}$, where $S_{k}=\partial T_{k}-\overline{B_{k}}$, each $T_{k}$ being the tent associated to the ball $B_{k}$.

Proof. For every $k \in \mathbb{N}$, let $\mu_{k}$ be the normalized 'surface' measure on the sphere $\partial B_{k}$, that is, $\mu_{k}\left(\partial B_{k}\right)=1$. An elementary computation with the Poisson kernel gives the estimate

$$
P\left[\mu_{k}\right](x, y) \geqslant A / y r_{k}^{n-1}
$$

if $(x, y) \in S_{k}$, for $k \in \mathbb{N}$, where $A$ is a positive constant depending only on the dimension $n$. Define a finite positive measure $\mu$ on $\mathbb{R}^{n}$ by the expression

$$
\mu=\frac{1}{A} \sum_{k} r_{k}^{\beta} \mu_{k}
$$

Then the function $b=P[\mu]$ satisfies the conditions required. Indeed, if $(x, y) \in S_{k}$ then

$$
b(x, y) \geqslant \frac{1}{A} r_{k}^{\beta} P\left[\mu_{k}\right](x, y) \geqslant \frac{r_{k}^{\beta-(n-1)}}{y} \geqslant \frac{1}{y^{n-\beta}} .
$$

Note that the condition $\beta \geqslant n-1$ has been used in the last inequality.

The fact that our proof of Theorem 2 only works for $\alpha \in[0,1]$ is a consequence of the restriction $\beta \in[n-1, n]$ in Lemma 2 .

Lemma 3. Let $u$ be subharmonic in the Carleson cylinder $C(0, h)$, bounded above by a positive constant $M$. Assume that $u^{*}(x) \leqslant 0$ for a.e. $x \in \mathbb{B}^{n}(0, h)$. Then, for every $\varepsilon \in(0,1)$ and $(x, y)$ with $|x| \leqslant \frac{1}{2} h, 0<y<h \varepsilon$, one has

$$
u(x, y) \leqslant A M \varepsilon
$$

where $A$ is a positive constant which depends only on $n$.

Proof. We may assume that $M=h=1$. To simplify notation we write here 
$B=B^{n}(0,1), C=C(0,1), D=\partial C-B$. Denote by $\omega(x, y)$ the harmonic measure of $D$ in the cylinder $C$, that is, the harmonic function in $C$ with boundary values +1 on $D$ and 0 on $B$. Then $u-\omega$ is a subharmonic function in the Lipschitz domain $C$ and it is bounded above there. Now, by Lemma D and the hypotheses, $u-\omega$ has non-positive normal limits a.e. on $\partial C$. Standard arguments show then that $u-\omega$ has a non-positive harmonic majorant and, consequently, $u-\omega \leqslant 0$ throughout $C$.

By symmetry and the Maximum Principle, we have

$$
\omega \leqslant v \quad \text { on } B^{n+1}(0,1) \cap \mathbb{R}_{+}^{n+1},
$$

where $v$ is the harmonic function in $B^{n+1}(0,1)$ with boundary values +1 on $\partial B^{n+1}(0,1) \cap \mathbb{R}_{+}^{n+1}$ and -1 on $\partial B^{n+1}(0,1) \cap \mathbb{R}^{n+1}$. The result follows from the inequalities above and the estimate

$$
|v(x, y)| \leqslant A y, \quad \text { where }|x| \leqslant \frac{1}{2},|y| \leqslant \frac{1}{2},
$$

which is easily derived from the explicit expression of $v$ in terms of the Poisson kernel of a ball.

COROllary. Let $u$ be a continuous subharmonic function in the Carleson cylinder $C(0, h)$ and let $\Omega$ be a component of $\{u>\lambda\}$ for some real number $\lambda$. Assume that the next two conditions are satisfied:

(i) $u \leqslant L$ in $\Omega \cap C(0, h)$ for some constant $L$;

(ii) $\left\{x \in B^{n}(0, h): u^{*}(x)\right.$ exists and $\left.u^{*}(x) \geqslant \lambda\right\}$ has zero measure.

Then, if $0<\varepsilon<1$, we have

$$
u(x, y) \leqslant \lambda+A \varepsilon(L-\lambda)
$$

for all $(x, y) \in \Omega$ with $x \in B^{n}\left(0, \frac{1}{2} h\right), 0<y \leqslant \varepsilon h$. Here $A$ is the constant of Lemma 3.

Proof. Define

$$
v= \begin{cases}u-\lambda & \text { in } \Omega, \\ 0 & \text { in } C(0, h) \backslash \Omega .\end{cases}
$$

Then $v$ is subharmonic in $C(0, h)$ and $0 \leqslant v \leqslant L-\lambda$ in $C(0, h)$. Then $v^{*}(x)$ exists for a.e. $x$ on $B^{n}(0, h)$ and therefore assumption (ii) implies in fact that $v^{*}(x)=0$ for a.e. $x \in B^{n}(0, h)$. Now, by Lemma 3 ,

$$
v(x, y) \leqslant A \varepsilon(L-\lambda)
$$

for all $(x, y)$ with $|x| \leqslant \frac{1}{2} h, 0<y<\varepsilon h$. Therefore

$$
u(x, y) \leqslant \lambda+A \varepsilon(L-\lambda)
$$

if $(x, y) \in \Omega,|x| \leqslant \frac{1}{2} h, 0<y<\varepsilon h$.

We remark that there is a version of this last corollary which is valid for discontinuous $u$; see $\S 6$. 


\section{Some harmonic measure estimates}

For a domain $\Omega$, a point $p_{0} \in \Omega$, and a subset $E$ of $\partial \Omega$, the harmonic measure of $E$ at $p_{0}$ in $\Omega$ will be denoted by $\omega\left(E, p_{0}, \Omega\right)$.

LEMMA 4. Given positive numbers $r$ and $h$, consider the cylinder $C(0, r, h)$ in $\mathbb{R}_{+}^{n+1}$. Then there exist positive constants $A, B$, depending only on $n$ such that

$$
\omega\left(\partial B^{n}(0, r) \times[0, h],(0, y), C(0, r, h)\right) \leqslant A \frac{y}{h} \exp \left(-B \frac{r}{h}\right), \quad \text { for } 0<y<h .
$$

Proof. Let $Q=\left\{x=\left(x_{1}, \ldots, x_{n}\right) \in \mathbb{R}^{n}:\left|x_{j}\right| \leqslant r / \vee_{n}, j=1, \ldots, n\right\}$ be the cube centred at the origin, inscribed in the ball $B^{n}(0, r)$. Then, by the Maximum Principle and the symmetry of the domains involved, we have the following chain of inequalities:

$$
\begin{aligned}
\omega\left(\partial B^{n}(0, r)\right. & \times[0, h],(0, y), C(0, r, h)) \\
& \leqslant \omega(\partial Q \times[0, h],(0, y), Q \times(0, h)) \\
& \leqslant 2^{n} \omega\left(\left\{\frac{r}{\sqrt{ } n}\right\} \times\left[-\frac{r}{\sqrt{ } n}, \frac{r}{\sqrt{ } n}\right]^{n-1} \times[0, h],(0, y), Q \times(0, h)\right) \\
& \leqslant 2^{n} \omega\left(\left\{\frac{r}{\sqrt{ } n}\right\} \times \mathbb{R}^{n-1} \times[0, h],(0, y),\left(-\infty, \frac{r}{\sqrt{ } n}\right) \times \mathbb{R}^{n-1} \times(0, h)\right) .
\end{aligned}
$$

Note now that this last harmonic measure does not depend on the variables $x_{2}, \ldots, x_{n}$, so it is equal to the following planar harmonic measure:

$$
\omega\left(\left\{\frac{r}{V_{n}}\right\} \times[0, h], i y,\left(-\infty, \frac{r}{\sqrt{ } n}\right) \times(0, h)\right) \leqslant A \frac{y}{h} \exp \left(-B \frac{r}{h}\right),
$$

the last inequality being easily checked by using conformal mapping of a half-strip.

We proceed now to the definition of the special domain that plays a relevant role in the proof of Theorem 1'.

Given $x_{0} \in \mathbb{R}^{n}, r>0$ and a sequence $\left(a_{k}\right)$ of positive numbers such that $\sum_{k=1}^{\infty} a_{k}=1$, put $s_{0}=0, s_{k}=\sum_{j=1}^{k} a_{j}$, and define a domain $\mathscr{D}$ in $\mathbb{R}_{+}^{n+1}$ as follows:

$$
\mathscr{D}=\left\{(x, y):\left|x-x_{0}\right|<r, 0<y<r \Phi\left(\frac{\left|x-x_{0}\right|}{r}\right)\right\}
$$

where

$$
\Phi(t)= \begin{cases}1 & \text { if } 0 \leqslant t<a_{1}=s_{1} \\ 2^{-k} & \text { if } s_{k} \leqslant t<s_{k+1}\end{cases}
$$

Lemma 5. Let $x_{0}, r$ and $\mathscr{D}$ be as above. For $k \in \mathbb{N}$, define

Then

$$
\Gamma_{k}=\partial \mathscr{D} \cap\left\{(x, y):\left|x-x_{0}\right| \geqslant r s_{k}\right\} \text {. }
$$

$$
\omega\left(\Gamma_{k},\left(x_{0}, y_{0}\right), \mathscr{D}\right) \leqslant A \exp \left(-B a_{k} 2^{k}\right), \text { for } 0<y_{0}<r,
$$

where $A, B$ are positive constants depending only on $n$. 
Proof. After a change of scale, if necessary, we may assume that $x_{0}=0$, and $r=1$. For $k \in \mathbb{N}$, define

$$
\begin{aligned}
& \Gamma_{k}^{\prime}=\left\{(x, y):|x|=s_{k}, 0 \leqslant y \leqslant 2^{-(k-1)}\right\}, \\
& \Gamma_{k}^{\prime \prime}=\left\{(x, y):|x|=\frac{1}{2}\left(s_{k-1}+s_{k}\right), 0 \leqslant y \leqslant 2^{-(k-1)}\right\} .
\end{aligned}
$$

By the Maximum principle,

$$
\omega\left(\Gamma_{k},\left(0, y_{0}\right), \mathscr{D}\right) \leqslant \omega\left(\Gamma_{k}^{\prime},\left(0, y_{0}\right), \mathscr{D}_{k}\right)
$$

where $\mathscr{D}_{k}=\mathscr{D} \cap\left\{(x, y):|x|<s_{k}\right\}$.

The Markov property of harmonic measure and the Maximum Principle give

$$
\omega\left(\Gamma_{k}^{\prime},\left(0, y_{0}\right), \mathscr{D}_{k}\right) \leqslant \omega\left(\Gamma_{k}^{\prime \prime},\left(0, y_{0}\right), G_{k}\right) \sup _{(x, y) \in \Gamma_{k}^{\prime \prime}} \omega\left(\Gamma_{k-1}^{\prime} \cup \Gamma_{k}^{\prime},(x, y), R_{k}\right)
$$

where

$$
\begin{aligned}
& G_{k}=\mathscr{D} \cap\left\{(x, y):|x|<\frac{1}{2}\left(s_{k-1}+s_{k}\right)\right\} \\
& R_{k}=\left\{(x, y): s_{k-1}<|x|<s_{k}, 0<y<2^{-(k-1)}\right\}
\end{aligned}
$$

In particular,

$$
\omega\left(\Gamma_{k},\left(0, y_{0}\right), \mathscr{D}\right) \leqslant \sup _{(x, y) \in \Gamma_{k}^{n}} \omega\left(\Gamma_{k-1}^{\prime} \cup \Gamma_{k}^{\prime},(x, y), R_{k}\right)
$$

Fix $(x, y) \in \Gamma_{k}^{\prime \prime}$ and recall that $a_{k}=s_{k}-s_{k-1}$. Then by Lemma 4 , we have

$$
\omega\left(\Gamma_{k-1}^{\prime} \cup \Gamma_{k}^{\prime},(x, y), R_{k}\right) \leqslant A \exp \left(-B a_{k} 2^{k}\right),
$$

as desired.

\section{Proof of Theorems $1^{\prime}$ and 2 for continuous subharmonic functions}

Suppose that $u$ is subharmonic in the Carleson cylinder $C(0,1)$, is continuous in $\bar{C}(0,1) \cap \mathbb{R}_{+}^{n+1}$, and satisfies the condition of Hornblower:

$$
\int_{0}^{1} \log ^{+} M(t) d t<\infty
$$

where $M(t)=\sup \{u(x, y):|x| \leqslant 1, t \leqslant y \leqslant 1\}$.

We shall construct, for every $r(0<r<1)$, a sequence of positive numbers $\left(a_{k}\right)$, where $\sum_{k} a_{k}=1$, as follows. First, choose an integer $m=m(r)$ such that

$$
\frac{1}{B}\left(\int_{0}^{r 2^{-m}} \log ^{+} M(t) d t+r \sum_{k=m+1}^{\infty} k 2^{-k}\right)<\frac{1}{2} r,
$$

where $B$ is the constant appearing in Lemma 5. Note that the boundedness of the integral $\left(\mathrm{H}^{\prime}\right)$ implies the convergence of the series

$$
\sum_{k=1}^{\infty} 2^{-k} \log ^{+} M\left(r 2^{-k}\right)
$$

and the above choice of $m$ implies as well that

$$
\frac{1}{B}\left(\sum_{k=m+1}^{\infty} 2^{-k} \log ^{+} M\left(r 2^{-k}\right)+\sum_{k=m+1}^{\infty} k 2^{-k}\right)<\frac{1}{2} \text {. }
$$


Now define the sequence $\left(a_{k}\right)$, depending on $r$, by

$$
a_{k}= \begin{cases}\frac{1}{B}\left(2^{-k} \log ^{+} M\left(r 2^{-k}\right)+k 2^{-k}\right) & \text { if } k \geqslant m+1, \\ \frac{1}{m}\left(1-\sum_{l=m+1}^{\infty} a_{l}\right) & \text { if } 1 \leqslant k \leqslant m .\end{cases}
$$

Observe that every $a_{k}$ is positive and that $\sum_{k} a_{k}=1$. We also define for convenience $a_{0}=0$.

The following lemma is fundamental in the proofs of Theorems 1 and 2 .

LeMma 6. Let $u$ be subharmonic in $C(0,1)$, continuous in $\bar{C}(0,1) \cap \mathbb{R}_{+}^{n+1}$ and satisfy condition $\left(\mathrm{H}^{\prime}\right)$ :

$$
\int_{0}^{1} \log ^{+} M(t) d t<\infty
$$

where $M(t)=\sup \left\{(u(x, y):|x| \leqslant 1, t \leqslant|y| \leqslant 1\}\right.$. Further, let $x_{0} \in \mathbb{B}^{n}$ and $h>0$ be such that $C\left(x_{0}, h\right) \subset C(0,1)$.

Then there exists a positive $\lambda_{0}$, depending only on $M=M(t)$ and $h$, such that the following holds: if both

(i) $\mid\left\{x \in B^{n}\left(x_{0}, h\right): u^{*}(x)\right.$ exists and $\left.u^{*}(x)>\frac{1}{2} \lambda\right\} \mid=0$, and

(ii) $u\left(x_{0}, y_{0}\right)>2 \lambda$,

are satisfied for some $\lambda>\lambda_{0}$ and $y_{0} \in\left(0, \frac{1}{4} h\right)$, and if $\Omega$ is the component of $\left\{u>\frac{1}{2} \lambda\right\} \cap C\left(x_{0}, h\right)$ containing $\left(x_{0}, y_{0}\right)$, then there exists $x \in \bar{B}^{n}\left(x_{0}, \frac{1}{2} h\right) \cap \partial \Omega$ so that $u$ is unbounded above in $C\left(x, \frac{1}{16} h\right) \cap \Omega$.

The proof will show that we can choose $\lambda_{0}(h)=2+A(m+1) M\left(\frac{1}{4} h 2^{-m}\right)$, where $A$ is the constant appearing in Lemma 5 and $m=m\left(\frac{1}{4} h\right)$ is the integer defined at the introduction of this section, corresponding to $r=\frac{1}{4} h$.

Proof. Suppose that the conclusion of the lemma does not hold. Then, either $\bar{B}^{n}\left(x_{0}, \frac{1}{2} h\right) \cap \partial \Omega=\varnothing$ or $u$ is bounded above in $C\left(x, \frac{1}{16} h\right) \cap \Omega$ for each $x \in$ $\bar{B}^{n}\left(x_{0}, \frac{1}{2} h\right) \cap \partial \Omega$. In either case, this implies the existence of a positive constant $L$ such that

$$
u \leqslant L \quad \text { on } C\left(x_{0}, \frac{1}{2} h\right) \cap \Omega .
$$

Let $r=\frac{1}{4} h$, and consider the domain $\mathscr{D}$ corresponding to $r, x_{0}$ and the sequence $\left(a_{k}\right)$ defined at the beginning of the section. Take a small positive number $\varepsilon$, and let $\Omega_{\varepsilon}$ be the component of $\Omega \cap \mathscr{D} \cap\left\{y>\frac{1}{4} \varepsilon h\right\}$ which contains $\left(x_{0}, y_{0}\right)$. Then the boundary of $\Omega_{\varepsilon}$ can be decomposed into three parts, say, $E, F, G$, such that

$$
\begin{aligned}
& E=\bigcup_{k=0}^{\infty} E_{k}, \quad \text { with } E_{k}=\partial \Omega_{\varepsilon} \cap\left\{s_{k} \leqslant 4\left|x-x_{0}\right| / h<s_{k+1}\right\} \cap \partial \mathscr{D}, \\
& F=\partial \Omega_{\varepsilon} \cap\left\{y=\frac{1}{4} \varepsilon h\right\}, \\
& G \subset \partial \Omega_{\varepsilon} \cap \partial \Omega .
\end{aligned}
$$


(Recall that $s_{k}=\sum_{j=1}^{k} a_{j}$ for every $k \in \mathbb{N}$ and $s_{0}=0$.) Note that $u \leqslant \frac{1}{2} \lambda$ on $G$ and, from the Corollary of Lemma 3 , deduce that

$$
u \leqslant \frac{1}{2} \lambda+C \varepsilon\left(L-\frac{1}{2} \lambda\right) \text { on } F .
$$

Since $\Omega_{\varepsilon} \subset \mathscr{D}$ and $E_{k} \subset \Gamma_{k}$, then by the Maximum principle and the estimate of Lemma 5 , we have that

$$
\omega\left(E_{k},\left(x_{0}, y_{0}\right), \Omega_{\varepsilon}\right) \leqslant \omega\left(\Gamma_{k},\left(x_{0}, y_{0}\right), \mathscr{D}\right) \leqslant A \exp \left(-B a_{k} 2^{k}\right),
$$

and therefore, harmonic majorization gives

$$
\begin{aligned}
2 \lambda<u\left(x_{0}, y_{0}\right) \leqslant & \frac{1}{2} \lambda+\frac{1}{2} \lambda+C \varepsilon\left(L-\frac{1}{2} \lambda\right)+A \sum_{k=0}^{\infty} M\left(\frac{1}{4} h 2^{-k}\right) \exp \left(-B a_{k} 2^{k}\right) \\
\leqslant & \lambda+C \varepsilon\left(L-\frac{1}{2} \lambda\right)+A \sum_{k=0}^{m} M\left(\frac{1}{4} h 2^{-k}\right) \\
& +A \sum_{k=m+1}^{\infty} \exp \left(\log ^{+} M\left(\frac{1}{4} h 2^{-k}\right)-B a_{k} 2^{k}\right) .
\end{aligned}
$$

Recalling the choice of the sequence $\left(a_{k}\right)$ we conclude that

$$
2 \lambda \leqslant \lambda+C \varepsilon\left(L-\frac{1}{2} \lambda\right)+A(m+1) M\left(\frac{1}{4} h 2^{-m}\right)+\sum_{k=m+1}^{\infty} e^{-k}
$$

and since $\varepsilon$ was arbitrary, we finally get

$$
2 \lambda \leqslant \lambda+A(m+1) M\left(\frac{1}{4} h 2^{-m}\right)+2,
$$

which contradicts the choice of $\lambda$. This contradiction completes the proof of the lemma.

Proof of Theorem 1'. Without loss of generality, we assume that $B=B^{n}\left(0, h_{0}\right)$ and $|F(u) \cap B|=0$, with $0<h_{0}<1$. As before, we let

$$
M(t)=\sup \{u(x, y):|x| \leqslant 1, t \leqslant y \leqslant 1\} .
$$

Let $h_{1}=\frac{1}{4} h_{0}$ and take $\lambda_{1}>2+A m_{1} M\left(\frac{1}{4} h_{1} 2^{-m_{1}}\right)$, where $A$ and $m_{1}=m\left(\frac{1}{4} h_{1}\right)$ have the same meanings as above.

Then $u$ must be unbounded above in the cylinder $C\left(0, \frac{1}{4} h_{1}\right)$ since otherwise $u$ would have finite vertical limits on a set of positive measure (Lemma $D$ ), contrary to the hypothesis. Therefore, a point $p_{1} \in C\left(0, \frac{1}{4} h_{1}\right)$ can be chosen so that $u\left(p_{1}\right)>2 \lambda_{1}$.

If $p_{1}=\left(x_{1}, y_{1}\right)$, then $C\left(x_{1}, h_{1}\right) \subset C\left(0, h_{0}\right)$ and $y_{1}<\frac{1}{4} h_{1}$. The argument follows in an inductive way. Assume that $p_{k}=\left(x_{k}, y_{k}\right), h_{k}, m_{k}=m\left(\frac{1}{4} h_{k}\right)$ and $\lambda_{k}$ have already been chosen so that the following conditions are satisfied:

$$
\begin{gathered}
u\left(p_{k}\right)>2 \lambda_{k}, \\
y_{k}<\frac{1}{4} h_{k}, \\
C\left(x_{k}, h_{k}\right) \subset C\left(x_{k-1}, h_{k-1}\right), \\
\lambda_{k}>2 \lambda_{k-1}+A m_{k} M\left(\frac{1}{4} h_{k} 2^{-m_{k}}\right) .
\end{gathered}
$$

Let $\Omega_{k}$ be the component of $\left\{u>\frac{1}{2} \lambda_{k}\right\} \cap C\left(x_{k}, h_{k}\right)$ which contains $p_{k}$. Then, by 
Lemma 6, there exists $x \in \bar{B}^{n}\left(x_{k}, \frac{1}{2} h_{k}\right) \cap \partial \Omega_{k}$ such that $u$ is unbounded above in $C\left(x, \frac{1}{16} h_{k}\right) \cap \Omega_{k}$. Put $h_{k+1}=\frac{1}{4} h_{k}$ and let $m_{k+1}=m\left(\frac{1}{4} h_{k}\right)$. If we choose $\lambda_{k+1}$ satisfying

$$
\lambda_{k+1}>2 \lambda_{k}+A m_{k+1} M\left(\frac{1}{4} h_{k+1} 2^{-m_{k+1}}\right),
$$

then there exists $p_{k+1} \in C\left(x, \frac{1}{16} h_{k}\right) \cap \Omega_{k}=C\left(x, \frac{1}{4} h_{k+1}\right) \cap \Omega_{k}$ such that

$$
u\left(p_{k+1}\right)>2 \lambda_{k+1} \text {. }
$$

Note that if $p_{k+1}=\left(x_{k+1}, y_{k+1}\right)$ then (3.1), (3.2), (3.3) and (3.4) hold for $k+1$ instead of $k$, so the iterative procedure works. It is clear that the sequence $\left\{\lambda_{k}\right\}$ constructed in this way tends to $+\infty$.

Since $p_{k}, p_{k+1}$ both belong to $\Omega_{k}$, which is a component of

$$
\left\{u>\frac{1}{2} \lambda_{k}\right\} \cap C\left(x_{k}, h_{k}\right) \text {, }
$$

they can be joined by a path $\gamma_{k}$ inside $\Omega_{k}$. In particular, $\left.u\right|_{\gamma_{k}}>\frac{1}{2} \lambda_{k}$. Define $\gamma=\bigcup_{k=1}^{\infty} \gamma_{k}$ to be the path obtained by connecting the successive paths $\gamma_{k}$. Since $h_{k+1}=\frac{1}{4} h_{k}$, and $C\left(x_{k+1}, h_{k+1}\right) \subset C\left(x_{k}, h_{k}\right)$ then $\left\{C\left(x_{k}, h_{k}\right)\right\}$ is a nested sequence of Carleson cylinders whose diameters tend to zero, so the path $\gamma$ certainly ends at a point $x_{0} \in B$. Since $\lambda_{k} \rightarrow \infty$, we have that $x_{0} \in A(u,+\infty)$. Theorem 1' is, therefore, completely proved.

Proof of Theorem 2. We can assume that

$$
u(x, y) \leqslant 1 / y^{\alpha}, \quad \text { where } 0<y \leqslant 1, \alpha \in[0,1],
$$

and also that $B$ is the unit ball of $\mathbb{R}^{n}$ and $|F(u) \cap B|=0$. Let $E=A(u,+\infty) \cap B$ and suppose $M_{n-\alpha}(E)<1=M_{n-\alpha}(B)$. Apply Lemma 1 to $E$, with $\beta=n-\alpha$, and obtain a sequence of balls $\left\{B_{k}\right\}$, each $B_{k}$ of radius $r_{k}$, such that

$$
\sum_{k} r_{k}^{\beta}<1, \quad\left|B \backslash \bigcup_{k} B_{k}\right|>0 \text {. }
$$

Since $\beta \in[n-1, n]$, Lemma 2 supplies a function $b$, positive and harmonic in $\mathbb{R}_{+}^{n+1}$ with

$$
b(x, y) \geqslant 2 / y^{\alpha}
$$

if $(x, y) \in \bigcup_{k} S_{k}$, where $S_{k}=\partial T_{k} \backslash \bar{B}_{k}$, each $T_{k}$ being the tent associated to the ball $B_{k}$, as defined in $\S 1$.

Consider the domain $P=C(0,1) \backslash \bigcup_{k} T_{k}$ and observe that

$$
\partial P \cap \mathbb{R}^{n} \cap A(u,+\infty)=\varnothing \quad \text { and } \quad\left|\partial P \cap \mathbb{R}^{n}\right|=\left|B \backslash \bigcup_{k} B_{k}\right|>0 \text {. }
$$

Define a function $v$ in $C(0,1)$ as

$$
v= \begin{cases}(u-b)^{+} & \text {in } P, \\ 0 & \text { in } C(0,1) \backslash P=\bigcup_{k} T_{k} .\end{cases}
$$

Then $v$ is continuous, subharmonic and positive in $C(0,1)$. Take $h_{1}>0$ such that

$$
\left|B^{n}\left(0,1-h_{1}\right) \backslash \bigcup_{k} B_{k}\right|>0 \text {. }
$$

We claim that $v$ is unbounded above in the cylinder $C\left(0,1-h_{1}, \frac{1}{4} h_{1}\right)$. 
Indeed, otherwise $u-b$ would be bounded above in the Lipschitz domain $P^{\prime}=P \cap C\left(0,1-h_{1}\right)$ and, consequently, $u-b$ would have vertical boundary values almost everywhere in $\partial P^{\prime} \cap \mathbb{R}^{n}$ (Lemma D) and, since $\left|\partial P^{\prime} \cap \mathbb{R}^{n}\right|>0$ and $b$ has vertical limits almost everywhere, we could conclude that $u$ would have finite vertical limits on a set of positive measure, contrary to the hypothesis. Therefore $v$ is unbounded above in $C\left(0,1-h_{1}, \frac{1}{4} h_{1}\right)$.

Because of the way $v$ has been defined, one has that

$$
\log ^{+} M(t, v) \leqslant \log ^{+} M(t, u),
$$

where $M(t, v)$ and $M(t, u)$ have the same meaning as above. In particular $v$ satisfies the Hornblower condition if $u$ does.

For the positive number $h_{1}$ selected before, let $m_{1}=m\left(\frac{1}{4} h_{1}\right)$ be the integer corresponding to $v$ and $r=\frac{1}{4} h_{1}$. Take $\lambda_{1}>2+A m_{1} M\left(\frac{1}{4} h_{1} 2^{-m_{1}}\right)$.

Choose $p_{1}=\left(x_{1}, y_{1}\right) \in C\left(0,1-h_{1}, \frac{1}{4} h_{1}\right)$. such that $u\left(p_{1}\right)>2 \lambda_{1}$. Then $C\left(x_{1}, h_{1}\right) \subset C(0,1)$ and $y_{1}<\frac{1}{4} h_{1}$. Successive applications of Lemma 6 to $v$ would furnish, in the same way as in the proof of Theorem 1, a path $\gamma$ ending at a point $x_{0} \in B$ such that $v$ tends to $+\infty$ along $\gamma$, so $x_{0} \in A(v,+\infty) \subset B \backslash \bigcup_{k} B_{k}$, and this is a contradiction, since $A(v,+\infty) \subset A(u,+\infty) \subset \bigcup_{k} B_{k}$. This contradiction, obtained from the assumption $M_{n-\alpha}(E)<1$, proves Theorem 2.

\section{A submartingale analogue of Theorem 2}

Given a probability space $(\Omega, \mathscr{F}, \mu)$ and an increasing sequence of $\sigma$-algebras of measurable sets $\left\{\mathscr{F}_{k}\right\}$, we say that $\left\{\mathscr{F}_{k}\right\}$ is regular if there exists $R>0$ such that

$$
\mu\left(C_{k}(A)\right) \leqslant R \mu(A)
$$

for each $A \in \mathscr{F}_{k+1}$ and all $k \in \mathbb{N}$, where $C_{k}(A)$ is the smallest $\mathscr{F}_{k}$-measurable cover of $A$ (see [6]). It is obvious that a $p$-adic filtration in the unit cube of $\mathbb{R}^{n}$ is regular.

For a submartingale $\mathscr{S}=\left(S_{k}, \mathscr{F}_{k}\right)$ in the probability space $(\Omega, \mathscr{F}, \mu)$ we define, as in the Introduction, the Fatou set of $\mathscr{S}, F(\mathscr{S})$, and the divergence set $A(\mathscr{S},+\infty)$ as

$$
\begin{aligned}
F(\mathscr{S}) & =\left\{\omega \in \Omega: \lim _{k} S_{k}(\omega) \text { exists and is finite }\right\}, \\
A(\mathscr{S},+\infty) & =\left\{\omega \in \Omega: \lim _{k} S_{k}(\omega)=+\infty\right\} .
\end{aligned}
$$

If $\mathscr{S}=\left(S_{k}, \mathscr{F}_{k}\right)$ is a submartingale then there exist a martingale $M=\left(M_{k}, \mathscr{F}_{k}\right)$ and a non-negative increasing sequence $\left(A_{k}\right)$ such that, for each $k \in \mathbb{N}, A_{k}$ is $\mathscr{F}_{k-1}$-measurable and

$$
S_{k}=M_{k}+A_{k} \text {. }
$$

This decomposition is unique [20] and it is called the Doob decomposition of the submartingale $\mathscr{S}$. Observe that $M_{k} \leqslant S_{k}$, so that

$$
A(M,+\infty) \subset A(S,+\infty) \text {. }
$$

The following lemma relates $F(M)$ and $F(\mathscr{Y})$. The set inclusions and set 
identities appearing in this lemma and its proof are understood to hold up to a set of $\mu$-measure zero.

LEMMA 7. Let $S=\left(S_{k}, \mathscr{F}_{k}\right)$ be a submartingale in the probability space $(\Omega, \mathscr{F}, \mu)$ and

$$
S_{k}=M_{k}+A_{k}
$$

be its Doob decomposition. If $\left\{\mathscr{F}_{k}\right\}$ is regular then

$$
F(M)=\left\{\omega \in \Omega: \inf _{k} M_{k}(\omega)>-\propto\right\} \subset F(\mathscr{S}) \cup A(\mathscr{S},+\infty) .
$$

Proof. Take $\omega \in \Omega$ so that $\left(M_{k}(\omega)\right)$ is bounded. Since $\left(A_{k}\right)$ is increasing, there are two possibilities:

(i) $\lim _{k} A_{k}(\omega)=+\infty$, and in this case $\lim _{k} S_{k}(\omega)=+\infty$ because of the boundedness of $\left(M_{k}(\omega)\right)$;

(ii) $\lim _{k} A_{k}(\omega)<+\infty$ and, consequently $\sup _{k} S_{k}(\omega)<+\infty$ for the same reason. Therefore

$$
\left\{\omega \in \Omega:\left(M_{k}(\omega)\right) \text { is bounded }\right\} \subset\left\{\omega: \sup _{k} S_{k}(\omega)<\propto\right\} \cup A(\mathscr{S},+\infty) .
$$

By a theorem of Chow (see the last corollary of [6]) we have that

$$
\left\{\omega: \sup _{k} S_{k}(\omega)<\infty\right\} \subset F(\mathscr{S})
$$

and

$$
\left\{\omega: \inf _{k} M_{k}(\omega)>-\infty\right\} \subset F(M),
$$

so that we have the following chain of inclusions:

$$
\begin{aligned}
F(M) & \subset\left\{\omega: \inf _{k} M_{k}(\omega)>-\infty\right\} \subset F(M) \\
& \subset\left\{\omega:\left(M_{k}(\omega)\right) \text { is bounded }\right\} \subset F(\mathscr{S}) \cup A(\mathscr{S},+\infty),
\end{aligned}
$$

as desired.

The inclusion $\left\{\sup _{k} S_{k}<\infty\right\} \subset F(\mathscr{S})$ is no longer true if no regularity condition is assumed (even if $\mathscr{S}$ is a martingale); see Example 2.8.2 in [22].

The following lemma describes how to positivize a martingale. It can be viewed as a discrete analogue of the so-called localization techniques in function theory, which are based on the study of the components of $\{u>\lambda\}$ when $u$ is a harmonic (or subharmonic) function.

LEMMA 8. Let $M_{k}=\sum_{j=0}^{k} X_{j}$ be a martingale, with respect to the increasing sequence of $\sigma$-algebras $\left\{\mathscr{F}_{k}\right\}$. Suppose that $M_{0} \geqslant 0$ and that, for each $k \in \mathbb{N}, \mathscr{F}_{k}$ is generated by a finite number of sets. 
Then there exists a martingale $T_{k}=\sum_{j=0}^{k} Y_{j}$ (with respect to the same $\sigma$-algebras) such that $T_{0}=M_{0}$ and that, for each $k \in \mathbb{N}$, the following statements hold:

(i) $0 \leqslant T_{k} \leqslant M_{k}^{+}$;

(ii) $\left|Y_{k}\right| \leqslant\left|X_{k}\right|$;

(iii) if $T_{k}(\omega)=0$ then $T_{j}(\omega)=0$ for all $j \geqslant k$.

Proof. To define the $T_{k}$ we use an inductive argument starting with $T_{0}=M_{0}$.

Fix $k \in \mathbb{N}$ and assume that, for $j=0,1, \ldots, k$, the $\mathscr{F}_{j}$-measurable functions $T_{j}=\sum_{m=0}^{j} Y_{m}$ have already been defined, satisfying (i), (ii), (iii) and also the martingale requirement about conditional expectation,

$$
E\left[Y_{j+1} / \mathscr{F}_{j}\right]=0, \quad \text { for } j=0,1, \ldots, k-1 .
$$

We will show that it is then possible to construct an $\mathscr{F}_{k+1}$-measurable function $T_{k+1}=T_{k}+Y_{k+1}$ satisfying (i), (ii) and the martingale condition

$$
E\left[Y_{k+1} / \mathscr{F}_{k}\right]=0 \text {. }
$$

Property (iii) will follow from the construction.

Since $T_{k}$ is given, defining $T_{k+1}$ is equivalent to defining $Y_{k+1}$. We claim that it possible to take

$$
Y_{k+1}=\lambda_{k+1} X_{k+1}
$$

for a certain $\mathscr{F}_{k+1}$-measurable function $\lambda_{k+1}$, to be defined below, with $0 \leqslant$ $\lambda_{k+1} \leqslant 1$.

Consider the following $\mathscr{F}_{k+1}$-events:

$$
\begin{aligned}
& A=\left\{T_{k}=0\right\}, \\
& B=\left\{T_{k}>0, T_{k}+X_{k+1} \leqslant 0\right\}, \\
& C=\left\{T_{k}>0, T_{k}+X_{k+1}>0, X_{k+1} \leqslant 0\right\}, \\
& D=\left\{T_{k}>0, X_{k+1}>0\right\},
\end{aligned}
$$

that form a partition of the whole space, since $T_{k} \geqslant 0$. Notice also that $A$ is $\mathscr{F}_{k}$-measurable, and that on $B$ one has $X_{k+1} \leqslant 0$.

In $A$, we set $\lambda_{k+1} \equiv 0$. In $B$ we would like to have

$$
T_{k}+Y_{k+1}=T_{k}+\lambda_{k+1} X_{k+1}=0,
$$

so we define

$$
\lambda_{k+1}=T_{k} /\left|X_{k+1}\right|
$$

Observe that $-X_{k+1}=\left|X_{k+1}\right| \geqslant T_{k}>0$ in $B$, so $0 \leqslant \lambda_{k+1} \leqslant 1$ in $B$.

In $C$ we take $\lambda_{k+1} \equiv 1$. Finally, we need to choose $\lambda_{k+1}$ in $D$ to ensure that

$$
E\left[\lambda_{k+1} X_{k+1} / \mathscr{F}_{k}\right]=0 .
$$

Notice that whatever the choice of $\lambda_{k+1}$ in $D$, with $0 \leqslant \lambda_{k+1} \leqslant 1$, statements (i), (ii), (iii) hold. Indeed:

in $A, T_{k}=0=T_{k+1}$;

in $B, T_{k+1}=T_{k}+\lambda_{k+1} X_{k+1}=0$;

in $C, 0<T_{k+1}=T_{k}+X_{k+1} \leqslant M_{k}+X_{k+1}=M_{k+1}=M_{k+1}^{+}$;

in $D, 0<T_{k+1}=T_{k}+\lambda_{k+1} X_{k+1} \leqslant M_{k}+X_{k+1}=M_{k+1}=M_{k+1}^{+}$. 
Suppose that $\mathscr{F}_{k}$ is generated by the sets $F_{1}, \ldots, F_{q}$ for some $q=q(k)$ and

$$
\mu\left(F_{i} \Delta F_{j}\right)=0(i \neq j), \mu\left(\Omega \backslash \bigcup_{i=1}^{a} F_{i}\right)=0 .
$$

Since $\left\{F_{1}, \ldots, F_{q}\right\}$ is a partition of $\Omega$, it is enough to define $\lambda_{k+1}$ as a constant value in each $F_{i} \cap D$; recall that $D$ is $\mathscr{F}_{k+1}$-measurable. For each $i$, with $1 \leqslant i \leqslant q$, take

$$
z^{i}= \begin{cases}0 & \text { if } \int_{F_{i} \cap D} X_{k+1}=0, \\ \frac{\int_{F_{i} \cap B} T_{k}+\int_{F_{i} \cap C}\left|X_{k+1}\right|}{\int_{F_{i} \cap D} X_{k+1}} & \text { if } \int_{F_{i} \cap D} X_{k+1}>0 .\end{cases}
$$

First we need to check that $0 \leqslant z^{i} \leqslant 1 \quad(1 \leqslant i \leqslant q)$. Indeed, since $\left(M_{k}\right)$ is a martingale, $E\left[X_{k+1} / \mathscr{F}_{k}\right]=0$ and this implies that

$$
0=\int_{F_{i}} X_{k+1}=\int_{F_{i} \cap A} X_{k+1}+\int_{F_{i} \cap B} X_{k+1}+\int_{F_{i} \cap C} X_{k+1}+\int_{F_{i} \cap D} X_{k+1} .
$$

The first term in the right-hand side is zero, because $F_{i} \cap A \in \mathscr{F}_{k}$. Therefore

$$
\int_{F_{i} \cap D} X_{k+1}=\int_{F_{i} \cap B}\left|X_{k+1}\right|+\int_{F_{i} \cap C}\left|X_{k+1}\right| \geqslant \int_{F_{i} \cap B} T_{k}+\int_{F_{i} \cap C}\left|X_{k+1}\right| .
$$

These inequalities show that $0 \leqslant z^{i} \leqslant 1$.

On the other hand,

$$
\begin{aligned}
\int_{F_{i}} \lambda_{k+1} X_{k+1} & =\int_{F_{i} \cap A} 0 \cdot X_{k+1}+\int_{F_{i} \cap B} \frac{T_{k}}{\left|X_{k+1}\right|} X_{k+1}+\int_{F_{i} \cap C} X_{k+1}+\int_{F_{i} \cap D} z^{i} X_{k+1} \\
& =-\int_{F_{i} \cap B} T_{k}-\int_{F_{i} \cap C}\left|X_{k+1}\right|+z^{i} \int_{F_{i} \cap D} X_{k+1} .
\end{aligned}
$$

If $\mu\left(F_{i} \cap D\right) \neq 0$ then the choice of $z^{i}$ makes this last expression equal to zero.

If $\mu\left(F_{i} \cap D\right)=0$ then $\int_{F_{i} \cap D} X_{k+1}=0$ and so $\int_{F_{i} \cap(B \cup C)} X_{k+1}=0$. But on $B \cup C$ one has that $X_{k+1} \leqslant 0$ and we conclude that $X_{k+1}=0, \mu$-a.e. on $B \cup C$. And finally, (4.1) shows that $\int_{F_{i}} \lambda_{k+1} X_{k+1}=0$.

The conclusion is that if we define

$$
\lambda_{k+1}=\frac{T_{k}}{\left|X_{k+1}\right|} \chi_{B}+\chi_{C}+\sum_{i=1}^{q} z^{i} \chi_{F_{i} \cap D}
$$

where $\chi_{E}$ denotes the characteristic function of $E$, then $\lambda_{k+1}$ is an $\mathscr{F}_{k+1}$ measurable function such that $0 \leqslant \lambda_{k+1} \leqslant 1$ and

$$
E\left[Y_{k+1} / \mathscr{F}_{k}\right]=0 \text {. }
$$

Therefore we have defined inductively the new martingale $\left(T_{k}\right)$ in such a way that (i), (ii) and (iii) hold for all $k \in \mathbb{N}$.

This completes the proof of the lemma.

Now we are ready to prove the analogue of Theorem 2 for martingales.

Proof of Theorem 3. Let $\left(S_{k}\right)$ be a p-adic submartingale in the unit cube $Q_{0}$ of 
$\mathbb{R}^{n}$. The probability space now is $Q_{0}$ endowed with its $n$-dimensional Lebesgue measure.

Suppose that $\left(S_{k}\right)$ satisfies the growth condition

$$
S_{k} \leqslant C p^{k \alpha} \text {, for all } k \in \mathbb{N},
$$

where $C>0$ and $\alpha \geqslant 0$. First we assume that $Q=Q_{0}$ and that

$$
|F(\mathscr{S})|=|A(\mathscr{S},+\infty)|=0 .
$$

We intend to prove that

$$
M_{n-\alpha}(A(\mathscr{S},+\infty)) \geqslant K
$$

for some positive constant $K$, depending only on $n$ and $p$.

Let $M=\left(M_{k}\right)$ be the martingale which appears in the Doob decomposition of $\mathscr{S}=\left(S_{k}\right)$. Then $M_{k} \leqslant S_{k}$ for all $k$ and Lemma 7 implies that

$$
\inf _{k} M_{k}(\omega)=-\infty
$$

for almost all $\omega \in Q_{0}$.

By adding a constant if necessary (recall that $M_{0}$ is constant in $Q_{0}$ ) we may assume that $M_{0}=0$. Take $\lambda>0$ and let $T=\left(T_{k}\right)$ be the martingale associated to $M+\lambda$ as in Lemma 8. Since $M_{k} \leqslant S_{k} \leqslant C p^{k \alpha}$, we have

$$
0 \leqslant T_{k} \leqslant\left(M_{k}+\lambda\right)^{+} \leqslant C p^{k \alpha}+\lambda .
$$

For every $p$-adic cube, $Q_{k}$, of the $k$-generation, define

$$
\mu\left(Q_{k}\right)=\int_{Q_{k}} T_{k}
$$

This choice defines a finite positive measure in $Q_{0}[\mathbf{2 0}$, p. 150]. Observe that $\mu\left(Q_{0}\right)=\lambda$, and that

$$
\mu\left(Q_{k}\right) /\left|Q_{k}\right|=\left.T_{k}\right|_{Q_{k}}
$$

if $Q_{k}$ is $p$-adic of the $k$ th generation. Now (4.2) and Lemma 8(iii) imply that $T_{k}$ is eventually 0 almost surely, and consequently $\mu$ is a singular measure.

Define

$$
A^{\prime}=\left\{x \in Q_{0}: D \mu(x)=+\infty\right\} .
$$

Thus if $x \in A^{\prime}$ and $\left(Q_{k}(x)\right)$ is the sequence of $p$-adic cubes that converges to $x$, then

$$
T_{k}(x)=\frac{\mu\left(Q_{k}(x)\right)}{\left|Q_{k}(x)\right|} \rightarrow \infty, \quad \text { as } k \rightarrow \infty
$$

In particular,

$$
A^{\prime} \subset A(T,+\infty) \subset A(M,+\infty) \subset A(\mathscr{S},+\infty) .
$$

If $Q_{k}$ is a $p$-adic cube of the $k$ th generation, then

$$
\mu\left(Q_{k}\right)=\int_{Q_{k}} T_{k} \leqslant\left(C p^{k \alpha}+\lambda\right)\left|Q_{k}\right| \leqslant(C+\lambda) s\left(Q_{k}\right)^{n-\alpha}
$$

where $s(Q)$ denotes the side length of the cube $Q$. 
Suppose that $\left\{Q_{j}\right\}$ is a covering of $A^{\prime}$ by $p$-adic cubes, contained in $Q_{0}$. Then

$$
\sum_{j} s\left(Q_{j}\right)^{n-\alpha} \geqslant \frac{1}{C+\lambda} \sum_{j} \mu\left(Q_{j}\right) \geqslant \frac{\mu\left(A^{\prime}\right)}{C+\lambda}=\frac{\lambda}{C+\lambda} .
$$

Since the $\beta$-content of a set defined with $p$-adic cubes is comparable to the $\beta$-content defined with balls, we have, by letting $\lambda$ tend to infinity, that

$$
M_{n-\alpha}\left(A^{\prime}\right)>K
$$

for some positive constant $K$, which depends only on $n$ and $p$.

In order to prove the local version of the result, we take a $p$-adic cube $Q$ of the generation $N$ contained in $Q_{0}$ and suppose that $|F(\mathscr{S}) \cap Q|=0$. By considering the martingale $\left(M_{k}+\lambda\right)_{k=N}^{\infty}$ restricted to $Q$, we see that the same argument above shows that, for each covering $\left(Q_{j}\right)$ of $A(\mathscr{Y},+\infty) \cap Q$ by $p$-adic cubes, we have

$$
\sum_{j}^{\infty} s\left(Q_{j}\right)^{n-\alpha} \geqslant s(Q)^{n-\alpha}
$$

and the result follows.

\section{Proof of Theorem 4}

As stated in the introduction, we deduce Theorem 4 from the following one-dimensional result.

THEOREM 4 '. For each $\alpha$, with $0<\alpha<1$, there exists a harmonic function $u$ in $\mathbb{R}_{+}^{2}$ such that $u \in \mathcal{M}_{\alpha}$, that $u$ has finite asymptotic limits at most in a countable set, and that

$$
\begin{aligned}
& \operatorname{dim} A(u,+\infty)=1-\alpha \quad \text { if } \alpha \leqslant \frac{1}{2}, \\
& 1-\alpha \leqslant \operatorname{dim} A(u,+\infty) \leqslant(1+2 \alpha)^{-1} \text { if } \alpha>\frac{1}{2} \text {. }
\end{aligned}
$$

To obtain Theorem 4 from Theorem $4^{\prime}$ it is enough to consider the function $v\left(x_{1}, x_{2}, \ldots, x_{n}, y\right)=u\left(x_{1}, y\right)$, where $u$ is the function in Theorem $4^{\prime}$.

The motivation for the proof of Theorem $4^{\prime}$ is the martingale construction which we briefly describe as follows.

Take $\alpha>0, R$ a large integer and $\delta$ a positive number such that $R^{-\alpha} \leqslant \delta<1$. For $t \in[0,1]$ define

$$
X_{0}(t)= \begin{cases}1 & \text { if } t \in[0, \delta] \\ -\delta(1-\delta)^{-1} & \text { if } t \in(\delta, 1]\end{cases}
$$

If $k \in \mathbb{N}$, define also

$$
X_{k}(t)=X_{0}\left(R^{k} t-\left[R^{k} t\right]\right)
$$

where $[\cdot]$ denotes the integer part. 
Now consider

$$
S_{n}(t)=\sum_{k=0}^{n} R^{k \alpha} X_{k}(t) .
$$

It is easy to check that $S_{n}(t)>0$ if and only if $X_{n}(t)>0$, for each $n \in \mathbb{N}$, so

$$
A(\mathscr{S},+\infty)=\bigcup_{n=1}^{\infty}\left\{t \in[0,1]: X_{k}(t)=+1, k=n, n+1, \ldots\right\}
$$

and a straightforward computation of the Hausdorff dimension finally gives that

$$
\operatorname{dim} A(\mathscr{S},+\infty)=1-(\log (1 / \delta))(\log R)^{-1} .
$$

Note that this value is exactly $1-\alpha$ if $\delta=R^{-\alpha}$. If, for each $k \in \mathbb{N}$, we define the interval families

$$
\begin{aligned}
& \mathscr{I}_{k}=\left\{\left[m R^{-k},(m+1) R^{-k}\right): \dot{m}=0,1, \ldots, R^{k}-1\right\}, \\
& \mathscr{I}_{k}^{+}=\left\{\left[m R^{-k},(m+\delta) R^{-k}\right): m=0,1, \ldots, R^{k}-1\right\}, \\
& \mathscr{I}_{k}^{-}=\left\{\left[(m+\delta) R^{-k},(m+1) R^{-k}\right): m=0,1, \ldots, R^{k}-1\right\} .
\end{aligned}
$$

Then

$$
A(\mathscr{S},+\infty)=\bigcup_{n=1}^{\infty} \bigcap_{k=n}^{\infty} \bigcup_{l \in \mathscr{I}_{k}^{+}} I .
$$

With this example in mind we proceed now to describe the construction needed in Theorem 4'.

(i) The starting point is to define, for each $k \in \mathbb{N}$, three families of intervals in $[0,1], \mathscr{I}_{k}, \mathscr{J}_{k}^{+}, \mathscr{J}_{k}^{-}$(later they can be extended periodically to the real line) that will play a similar role to the three interval families defined above.

Suppose that two increasing sequences of positive integers $\left(p_{k}\right),\left(q_{k}\right)$ and a decreasing sequence of positive numbers $\left(\delta_{k}\right)$ have already been chosen (the exact choices and their dependences will be made explicit later). The main properties that we require from these interval families are that, for each $k \in \mathbb{N}$, $\mathscr{F}_{k}^{+}$and $\mathscr{F}_{k}^{-}$are mutually disjoint, form a partition of $[0,1]$ and, for every $J \in \mathscr{J}_{k}$, we have $J=J^{+} \cup J^{-}$, where $J^{+} \in \mathscr{J}_{k}^{+}, J^{-} \in \mathscr{J}_{k}^{-}, J^{+} \cap J^{-}=\varnothing$ and

$$
\left|J^{+}\right|=\delta_{k}|J|, \quad\left|J^{-}\right|=\left(1-\delta_{k}\right)|J| .
$$

The families $\mathscr{I}_{k}, \mathscr{I}_{k}^{+}, \mathscr{I}_{k}^{-}$can be constructed inductively. Define $\mathscr{J}_{1}=\{[0,1]\}$, $\mathscr{J}_{1}^{+}=\left\{\left[0, \delta_{1}\right)\right\}, \mathscr{J}_{1}^{-}=\left\{\left[\delta_{1}, 1\right]\right\}$. Suppose that $\mathscr{J}_{m}, \mathscr{J}_{m}^{ \pm}$, for $m=1,2, \ldots, k-1$, have already been defined. Take $J \in \mathscr{I}_{k-1}$, and suppose that $J=J^{+} \cup J^{-}$, where $J^{+} \cup J^{-}=\varnothing, J^{+} \in \mathscr{J}_{k-1}^{+}, J^{-} \in \mathscr{J}_{k-1}^{-}$and

$$
\left|J^{+}\right|=\delta_{k-1}|J|, \quad\left|J^{-}\right|=\left(1-\delta_{k-1}\right)|J| .
$$

We will separately define the intervals of $\mathscr{I}_{k}$ contained in $\mathrm{J}^{+}$and $\mathrm{J}^{-}$.

Divide $J^{+}$into infinitely many intervals which are determined by the partition $\left(x_{n}\right)_{-\infty}^{\infty}$, where $x_{0}$ is the middle point of $J^{+}, x_{n}=x_{n-1}+2^{-(n+1)}\left|J^{+}\right|$if $n>0$, and $x_{n}=x_{0}-\left(x_{-n}-x_{0}\right)$, if $n<0$. Denote by $\left(J_{n}\right)_{-\infty}^{\infty}$ the partition of $J^{+}$obtained in this way. Observe that $\left|J_{n}\right|=2^{-(n+1)}\left|J^{+}\right|$. (This infinite subdivision is used by Berman in [5].) Now, for every $n \in \mathbb{Z}$, divide $J_{n}$ into $n^{2} p_{k}$ intervals of the same length, say $\left\{J_{n, j}: j=1,2, \ldots, n^{2} p_{k}\right\}$ and, for each $J_{n, j}$, divide it again into two intervals $J_{n, j}^{+}$and 
$J_{n, l}^{-}$such that the left (right) endpoint of $J_{n, j}^{+}\left(J_{n, j}^{-}\right)$is the left (right) endpoint of $J_{n, j}$ and lengths are related by the rule

$$
\left|J_{n, j}^{+}\right|=\delta_{k}\left|J_{n, j}\right|, \quad\left|J_{n, j}^{-}\right|=\left(1-\delta_{k}\right)\left|J_{n, j}\right| .
$$

Then we define

$$
\begin{aligned}
& \mathscr{J}_{k} \cap J^{+}=\left\{J_{n, j}: n \in \mathbb{Z}, j=1,2, \ldots, n^{2} p_{k}\right\}, \\
& \mathscr{J}_{k}^{+} \cap J^{+}=\left\{J_{n, j}^{+}: n \in \mathbb{Z}, j=1,2, \ldots, n^{2} p_{k}\right\}, \\
& \mathscr{J}_{k}^{-} \cap J^{+}=\left\{J_{n, j}^{-}: n \in \mathbb{Z}, j=1,2, \ldots, n^{2} p_{k}\right\} .
\end{aligned}
$$

In order to define the intervals of $\mathscr{J}_{k}, \mathscr{J}_{k}^{+}, \mathscr{J}_{k}^{-}$which are contained in $J^{-}$we repeat the above procedure but substituting $p_{k}$ by $q_{k}$. If we now write $\left(I_{m, i}\right)$, $\left(I_{m . i}^{+}\right),\left(I_{m, i}^{-}\right)$, where $m \in \mathbb{Z}$ and $i=1,2, \ldots, m^{2} q_{k}$, for the corresponding intervals, then

$$
\begin{aligned}
& \mathscr{J}_{k} \cap J^{-}=\left\{I_{m, i}: m \in \mathbb{Z}, i=1,2, \ldots, m^{2} q_{k}\right\}, \\
& \mathscr{J}_{k}^{+} \cap J^{-}=\left\{I_{m, i}^{+}: m \in \mathbb{Z}, i=1,2, \ldots, m^{2} q_{k}\right\}, \\
& \mathscr{I}_{k}^{-} \cap J^{-}=\left\{I_{m, i}^{-}: m \in \mathbb{Z}, i=1,2, \ldots, m^{2} q_{k}\right\} .
\end{aligned}
$$

Since this construction can be carried out for every $J \in \mathscr{I}_{k-1}$, we have inductively defined the families $\mathscr{I}_{k}, \mathscr{I}_{k}^{+}, \mathscr{J}_{k}^{-}$. By periodic extension we assume that these families are defined in the whole real line. Observe that $\mathscr{F}_{k}^{+}, \mathscr{J}_{k}^{-}$form a partition of $\mathbb{R}$, for every $k \in \mathbb{N}$. Furthermore, if $J \in \mathscr{I}_{k-1}$ and $J=J^{+} \cup J^{-}$as above, then the length of each $J_{n, j} \in \mathscr{J}_{k} \cap J^{+}$is

$$
\left|J_{n, j}\right|=\left(n^{2} 2^{n+1} p_{k}\right)^{-1}\left|J^{+}\right|=\left(n^{2} 2^{n+1} p_{k}\right)^{-1} \delta_{k-1}|J|
$$

and the length of each $I_{m, i} \in \mathscr{I}_{k} \cap J^{-}$is

$$
\left|I_{m, i}\right|=\left(m^{2} 2^{m+1} q_{k}\right)^{-1}\left|J^{-}\right|=\left(m^{2} 2^{m+1} q_{k}\right)^{-1}\left(1-\delta_{k-1}\right)|J| .
$$

In order to get homogeneous bounds we assume hereafter that the condition

$$
p_{k} \geqslant \delta_{k-1} q_{k}
$$

holds for all $k \in \mathbb{N}$. Then the length of each interval in $\mathscr{J}_{k}$ is at most $\left(q_{1} q_{2} \ldots q_{k}\right)^{-1}$ and the length of each interval in $\mathscr{J}_{k}^{+}$is at most $\delta_{k}\left(q_{1} q_{2} \ldots q_{k}\right)^{-1}$.

(ii) For every $k \in \mathbb{N}$, define

$$
h_{k}= \begin{cases}+1 & \text { on the intervals of } \mathscr{J}_{k}^{+}, \\ -\delta_{k}\left(1-\delta_{k}\right)^{-1} & \text { on the intervals of } \mathscr{J}_{k}^{-}\end{cases}
$$

Note that each $h_{k}$ is a well-defined bounded step function on $\mathbb{R}$. We also denote by $h_{k}$ its harmonic extension to the upper half plane.

If $k \in \mathbb{N}$, define, for $0 \leqslant t \leqslant 1$, the indefinite integral

$$
H_{k}(t)=\int_{0}^{t} h_{k}(s) d s
$$

Since $h_{k}$ is periodic, of period 1 , and has zero mean on $[0,1]$ (in fact $h_{k}$ has zero 
mean on each interval of $\mathscr{F}_{k}$ ), then $H_{k}$ can also be extended periodically to the real line. Integration by parts gives

$$
h_{k}(x+i y)=-\int_{-\infty}^{+\infty} P_{t}(t-x, y) H_{k}(t) d t,
$$

where $P(t, y)=y\left(t^{2}+y^{2}\right)^{-1}$ is the non-normalized Poisson kernel in the upper half plane and $P_{t}$ denotes partial differentiation with respect to $t$.

The following lemma collects some of the boundedness properties of the functions $h_{k}, H_{k}$ that will be needed later.

Lemma 9. Let $k \in \mathbb{N}$.

(i) The following hold:

$$
\max _{J \in \mathscr{I}_{k}}|J| \leqslant\left(q_{1} q_{2} \ldots q_{k}\right)^{-1}, \max _{J \in \mathscr{I}_{k}^{+}}|J| \leqslant \delta_{k}\left(q_{1} q_{2} \ldots q_{k}\right)^{-1} .
$$

Furthermore, if $J \in \mathscr{J}_{k}$, then

$$
\max _{J}\left|H_{k}\right| \leqslant \delta_{k}|J|
$$

In particular,

$$
\max \left|H_{k}\right| \leqslant \delta_{k}\left(q_{1} q_{2} \ldots q_{k}\right)^{-1} .
$$

(ii) If $z=x+i y \in \mathbb{R}_{+}^{2}$, then

$$
\left|h_{k}(z)\right| \leqslant 2 \delta_{k} y^{-1}\left(q_{1} q_{2} \ldots q_{k}\right)^{-1} .
$$

(iii) If $J \in \mathscr{J}_{k}^{+} \cup J_{k}^{-}$and $\left(J_{n}\right)_{-\infty}^{+\infty}$ is its subsequent infinite subdivision, then for all $j>k$,

$$
\max _{J_{n}}\left|H_{j}\right| \leqslant \begin{cases}\delta_{j} \delta_{k}^{-1}\left(q_{k+1} q_{k+2} \ldots q_{j}\right)^{-1}\left|J_{n}\right| & \text { if } J \in \mathscr{I}_{k}^{+}, \\ \delta_{j}\left(q_{k+1} q_{k+2} \ldots q_{j}\right)^{-1}\left|J_{n}\right| & \text { if } J \in \mathscr{I}_{k}^{-}\end{cases}
$$

Proof. The first two inequalities in (i) have already been stated, as consequences of assumption (5.1), and (5.3) easily follows from the definition of $H_{k}$. Property (ii) is deduced from (i) and representation (5.2), since

$$
\left|h_{k}(x)\right| \leqslant \max \left|H_{k}\right| \int_{-\infty}^{+\infty}\left|P_{t}(t-x, y)\right| d t=2 y^{-1} \delta_{k}\left(q_{1} q_{2} \ldots q_{k}\right)^{-1} .
$$

Finally (iii) follows from assumption (5.1) together with the fact that the length of each subinterval of $J_{n}$ in $\mathscr{J}_{j}$ is, at most, $\left|J_{n}\right|\left(p_{k+1} q_{k+2} \ldots q_{j}\right)^{-1}$ whenever $J \in \mathscr{J}_{k}^{+}$, and is, at most $\left|J_{n}\right|\left(q_{k+1} q_{k+2} \ldots q_{j}\right)^{-1}$ whenever $J \in \mathscr{J}_{k}^{-}$.

The key estimate in the proof of Theorem $4^{\prime}$ is provided by the following lemma.

Lemma 10. Let $k \in \mathbb{N}$ and $I \in \mathscr{I}_{k}^{-}, J \in \mathscr{I}_{k}^{+}$. Denote by $C(I)$ and $C(J)$ the arcs of the circles which pass through the endpoints of I and J, respectively, and intersect I and $J$ with angles $\frac{1}{2} \delta_{k}$ and $\frac{1}{4} \pi$, respectively. Then, for all $j>k$, the inequality

$$
\left|h_{j}(z)\right| \leqslant A \delta_{j} \delta_{k}^{-1}\left(q_{k+1} q_{k+2} \ldots q_{j}\right)^{-1}
$$


holds for every $z \in C(I)$ or $z \in C(J)$ and some absolute constant $A>1$.

Proof. Let $I=(a, b) \in \mathscr{J}_{k}^{-}$. From (5.2) we deduce that

$$
\begin{aligned}
\left|h_{j}(x+i y)\right| \leqslant & \left|\int_{-\infty}^{a} P_{t}(t-x, y) H_{j}(t) d t\right|+\left|\int_{a}^{b} P_{t}(t-x, y) H_{j}(t) d t\right| \\
& +\left|\int_{b}^{+\infty} P_{t}(t-x, y) H_{j}(t) d t\right| .
\end{aligned}
$$

We estimate these three integrals separately. The boundedness of the third integral is completely parallel to the first one, so we restrict our attention to the first two integrals.

(i) To estimate the first integral in (5.4), suppose first that $L \in \mathscr{J}_{k}^{ \pm}$with $L \subset(-\infty, a)$ and let $\left(L_{n}\right)_{-\infty}^{+\infty}$ be its infinite subdivision, as described in the construction of the interval families. By the previous lemma, we have

$$
\begin{aligned}
\left|\int_{L} P_{t}(t-x, y) H_{j}(t) d t\right| & \leqslant \delta_{j} \delta_{k}^{-1}\left(q_{k+1} q_{k+2} \ldots q_{j}\right)^{-1} \sum_{-\infty}^{+\infty}\left|L_{n}\right| \int_{L_{n}}\left|P_{t}(t-x, y)\right| d t \\
& \leqslant 2 \delta_{j} \delta_{k}^{-1}\left(q_{k+1} q_{k+2} \ldots q_{j}\right)^{-1} \sum_{-\infty}^{+\infty} \int_{L_{n+1}} P(x-t, y) d t .
\end{aligned}
$$

Summing over all intervals $L$ we finally obtain

$$
\begin{aligned}
\left|\int_{-\infty}^{a} P_{t}(t-x, y) H_{j}(t) d t\right| & \leqslant 2 \delta_{j} \delta_{k}^{-1}\left(q_{k+1} q_{k+2} \ldots q_{j}\right)^{-1} \int_{-\infty}^{a} P(x-t, y) d t \\
& \leqslant(2 / \pi) \delta_{j} \delta_{k}^{-1}\left(q_{k+1} q_{k+2} \ldots q_{j}\right)^{-1} .
\end{aligned}
$$

(ii) To estimate the second integral in (5.4) we also consider the infinite subdivision $\left(I_{n}\right)_{-\infty}^{+\infty}$ of $I=(a, b)$. Suppose that $x \in I_{N}$ and let $I_{N-1}, I_{N+1}$ be the left and right adjacent intervals to $I_{N}$. We divide the integral into three parts:

$$
\begin{aligned}
\int_{a}^{b} P_{t}(t-x, y) H_{j}(t) d t= & \sum_{-\infty}^{N-2} \int_{I_{n}} P_{t}(t-x, y) H_{j}(t) d t \\
& +\int_{I_{N-1} \cup I_{N} \cup I_{N+1}} P_{t}(t-x, y) H_{j}(t) d t \\
& +\sum_{N+2}^{\infty} \int_{I_{n}} P_{t}(t-x, y) H_{j}(t) d t .
\end{aligned}
$$

By the previous lemma and computations similar to those given in (i), the first and the third terms in the right-hand part of (5.5) are bounded by $(2 / \pi) \delta_{j}\left(q_{k+1} q_{k+2} \ldots q_{j}\right)^{-1}$. To deal with the second term we again use Lemma 9 and conclude that

$$
\begin{aligned}
\mid \int_{I_{N-1} \cup I_{N} \cup I_{N+1}} P_{t}(t-x, y) & H_{j}(t) d t \mid \\
& \leqslant 2\left|I_{N}\right| \delta_{j}\left(q_{k+1} q_{k+2} \ldots q_{j}\right)^{-1} \int_{I_{N-1} \cup I_{N} \cup I_{N+1}}\left|P_{t}(t-x, y)\right| d t \\
& \leqslant 4\left|I_{N}\right| y^{-1} \delta_{j}\left(q_{k+1} q_{k+2} \ldots q_{j}\right)^{-1} .
\end{aligned}
$$


Now a simple geometric argument shows that $\left|I_{N}\right| y^{-1} \leqslant A \delta_{k}^{-1}$ whenever $z=$ $x+i y \in C(I)$, and putting together the conclusions of (i), (ii) we get

$$
\left|h_{j}(z)\right| \leqslant A \delta_{j} \delta_{k}^{-1}\left(q_{k+1} q_{k+2} \ldots q_{j}\right)^{-1}
$$

if $z \in C(I)$, as desired.

The case $J \in \mathscr{I}_{k}^{+}$follows with minor changes: now the first and third integrals in (5.5) are bounded by

$$
(2 / \pi) \delta_{j} \delta_{k}^{-1}\left(q_{k+1} q_{k+2} \ldots q_{j}\right)^{-1},
$$

but the ratio $\left|I_{N}\right| y^{-1}$ is bounded by an absolute constant, so the result follows.

At this point we can define the function appearing in Theorem 4'. First, we make some choices. Let $\alpha>0$, let $R$ be a large integer (we will require for instance $R^{\alpha} \geqslant 2$ ), and let $\left(n_{k}\right)$ be an increasing sequence of positive integers satisfying

$$
n_{k+1}-n_{k} \geqslant 2 \alpha\left(n_{k}-n_{k-1}\right)
$$

for each $k \in \mathbb{N}$. If $k \in \mathbb{N}$, put

$$
\begin{aligned}
& q_{k}=R^{n_{k}-n_{k-1},} \\
& \delta_{k}=16 A R^{-\alpha\left(n_{k}-n_{k-1}\right)}, \\
& p_{k}=\left[\delta_{k-1} q_{k}\right]+1,
\end{aligned}
$$

where $A$ is the constant in Lemma 10 .

We define, for $z=x+i y \in \mathbb{R}_{+}^{2}$,

$$
u(z)=\sum_{k=1}^{\infty} R^{\alpha n_{k}} h_{k}(z)
$$

Lemma 11. Let $k \in \mathbb{N}$ and $J \in \mathscr{J}_{k}^{+} \cup \mathscr{F}_{k}^{-}$. Under the assumptions above we have

$$
\left|u(z)-R^{\alpha n_{k}} h_{k}(z)\right| \leqslant \frac{1}{4} \delta_{k} R^{\alpha n_{k}}
$$

if $z \in C(J)$.

Proof. Substituting the expression for $u(z)$ and using Lemma 10 together with condition (5.6), we obtain the following chain of inequalities:

$$
\begin{aligned}
\left|u(z)-R^{\alpha n_{k}} h_{k}(z)\right| & \leqslant R^{\alpha n_{k}}\left(R^{-\alpha\left(n_{k}-n_{k-1}\right)}+A R^{n_{k}-\alpha n_{k-1}} \sum_{j=k+1}^{\infty} R^{-\left(n_{j}-\alpha n_{j-1}\right)}\right) \\
& \leqslant 2 A R^{\alpha n_{k}}\left(R^{-\alpha\left(n_{k}-n_{k-1}\right)}+R^{-\left[n_{k+1}-n_{k}-\alpha\left(n_{k}-n_{k-1}\right)\right]}\right) \\
& \leqslant 4 A R^{\alpha n_{k}+\alpha\left(n_{k}-n_{k-1}\right)}=\frac{1}{4} \delta_{k} R^{\alpha n_{k}} .
\end{aligned}
$$

Now we can prove Theorem 4'. 
Proof of Theorem 4'. First we show that $u \in M_{\alpha}$.

Take $y>0$ and select $k \in \mathbb{N}$ so that $R^{-n_{k}} \leqslant y<R^{-n_{k-1}}$. If we divide the series which defines $u(x+i y)$ into two parts and use Lemma 9(ii) we obtain

$$
\begin{aligned}
u(x+i y) & \leqslant \sum_{j=1}^{k-1} R^{\alpha n_{j}}+2 y^{-1} \sum_{j=k}^{\infty} R^{\alpha n_{j}} \delta_{j}\left(q_{1} q_{2} \ldots q_{j}\right)^{-1} \\
& \leqslant A\left(R^{\alpha n_{k-1}}+y^{-1} \delta_{k} R^{-(1-\alpha) n_{k}}\right) \leqslant A y^{-\alpha}
\end{aligned}
$$

so $u \in \mathcal{M}_{\alpha}$.

Now we prove that $u$ has finite asymptotic limits in at most a countable set of $\mathbb{R}$.

For each $k \in \mathbb{N}$, denote by $N_{k}$ the set of all endpoints of the intervals of $\mathscr{F}_{k}^{+}$and $\mathscr{F}_{k}^{-}$. Then $N=\bigcup_{k} N_{k}$ is countable.

Let $x \in \mathbb{P}-N$ and suppose that $J_{k}$ is the interval of $\mathscr{I}_{k}^{+} \cup \mathscr{F}_{k}^{-}$which contains $x$ for $k \in \mathbb{N}$. It is easy to check that if $z \in C\left(J_{k}\right)$ then the harmonic measure of $J_{k}$ from $z$ is $1-\left(\delta_{k} / 2 \pi\right)$ if $J_{k} \in \mathscr{J}_{k}^{-}$, and $\frac{3}{4}$ if $J_{k} \in \mathscr{J}_{k}^{+}$, so by harmonic majorization we have

$$
\begin{array}{ll}
h_{k}(z) \leqslant-\delta_{k}\left(1-\delta_{k}\right)^{-1}\left(1-\frac{\delta_{k}}{2 \pi}\right)+\frac{\delta_{k}}{2 \pi} \leqslant-\frac{1}{2} \delta_{k} & \text { if } J_{k} \in \mathscr{J}_{k}^{-}, \\
h_{k}(z) \geqslant \frac{3}{4}-\frac{1}{4} \delta_{k}\left(1-\delta_{k}\right)^{-1} \geqslant \frac{1}{2} & \text { if } J_{k} \in \mathscr{J}_{k}^{+} .
\end{array}
$$

Lemma 11 then shows that

$$
\begin{array}{ll}
u(z) \leqslant-\frac{1}{4} \delta_{k} R^{\alpha n_{k}}=-4 A R^{\alpha n_{k-1}} & \text { if } J_{k} \in \mathscr{J}_{k}^{-}, \\
u(z) \geqslant \frac{1}{4} R^{\alpha n_{k}} & \text { if } J_{k} \in \mathscr{J}_{k}^{+},
\end{array}
$$

whenever $z \in C\left(J_{k}\right)$. In particular, $u$ cannot have a finite asymptotic limit at $x$, as desired.

Finally we prove the statements concerning the dimension of $A(u,+\infty)$. Inequality (5.7) shows that if $u$ has asymptotic limit $+\infty$ at a point $x \in \mathbb{R}$, then the set of integers $k$ such that $x$ belongs to some interval of $\mathscr{J}_{k}^{-}$must be finite. Equivalently,

$$
A(u,+\infty) \subset \bigcup_{k=1}^{\infty} \bigcap_{j=k}^{\infty} \bigcup_{J \in \mathscr{I}_{k}^{+}} J .
$$

The values of the Hausdorff dimension that we obtain are a consequence of the following technical lemma, whose proof is omitted.

LEMMA 12. Let $\left(n_{k}\right)$ be an increasing sequence of positive integers satisfying

$$
n_{k} / n_{k-1} \rightarrow L, \quad n_{k} / k \rightarrow \infty
$$

as $k \rightarrow \infty$, where $L \in[1, \infty)$. Then, if $E$ is the set in the right-hand side of (5.8), we have

$$
\operatorname{dim} E \leqslant(L-\alpha)(L-\alpha+\alpha L)^{-1}
$$

To end the proof of Theorem 4', it is convenient to distinguish the cases $\alpha \leqslant \frac{1}{2}$ and $\alpha>\frac{1}{2}$.

(i) If $0<\alpha \leqslant \frac{1}{2}$, we choose $n_{k}=k^{3}$. Then $L=1$ and condition (5.6) is satisfied. The conclusion is that $\operatorname{dim} E \leqslant 1-\alpha$. 
(ii) If $\alpha>\frac{1}{2}$, then condition (5.6) forces $L \geqslant 2 \alpha$. In this case we may choose $n_{k}=k(2 \alpha)^{k}$, for which $L=2 \alpha$, and we obtain from the last lemma that

Therefore,

$$
\operatorname{dim} E \leqslant(1+2 \alpha)^{-1} \text {. }
$$

$$
\operatorname{dim} A(u,+\infty) \leqslant \begin{cases}1-\alpha & \text { if } 0<\alpha \leqslant \frac{1}{2}, \\ (1+2 \alpha)^{-1} & \text { if } \alpha>\frac{1}{2},\end{cases}
$$

and Theorem $4^{\prime}$ is completely proved.

\section{The discontinuous case}

In this section we discuss what changes are required in the proofs of Theorems $1^{\prime}$ and 2 to make them cover the discontinuous case as well. It turns out that the same ideas will work; we only need to switch to the language of fine potential theory.

Recall that the fine topology in $\mathbb{R}^{n+1}$, or in any subdomain of it, is the coarsest topology making all subharmonic functions continuous. We shall use the terminology 'finely open' or 'fine boundary' to describe sets with respect to the fine topology. Of course, every Euclidean open set is finely open.

First we observe that in the proof of Lemma 3 no continuity of $u$ is needed.

LEMMA 13. The corollary to Lemma 3 remains true for a discontinuous $u$ when $\Omega$ is a fine component of the finely open set $\{u>\lambda\}$.

Notice that it is true, although non-trivial to prove, that fine components of finely open sets are finely open $[9,10]$.

Now the proof in $\S 1$ applies verbatim once we observe that the function $v$ defined by

$$
v= \begin{cases}u-\lambda & \text { in } \Omega, \\ 0 & \text { in } C(0, h) \backslash \Omega,\end{cases}
$$

is subharmonic in $C(0, h)$; see [9, Lemma 10.1 and Theorem 9.8] or [14, Lemma 4.5].

The next place where continuity is used is Lemma 6 in $\S 3$.

Lemma 14. Lemma 6 remains true for a discontinuous $u$ when $\Omega$ is the fine component of $\left\{u>\frac{1}{2} \lambda\right\} \cap C\left(x_{0}, h\right)$ containing $\left(x_{0}, y_{0}\right)$.

The proof of Lemma 14 is again similar to that of Lemma 6. We now choose $\Omega_{\varepsilon}$ to be the fine component of $\Omega \cap \mathscr{D} \cap\left\{y>\frac{1}{4} \varepsilon h\right\}$ which contains $\left(x_{0}, y_{0}\right)$, and decompose the fine boundary of $\Omega_{\varepsilon}$ into three sets $E, F$ and $G$ as in $\S 3$. The fine potential theory allows similar use of the Maximum Principle and harmonic majorization as in the proof of Lemma 6 [9] and we conclude that Lemma 14 is proved.

Finally, after this preparation, to prove Theorems $1^{\prime}$ and 2 in the discontinuous case we only need to invoke the fact from fine potential theory that fine components of finely open sets are pathwise connected $[19,16,10]$. Then the 
proofs in $\S 3$ apply after obvious changes, and we conclude that Theorems $1^{\prime}$ and 2 are completely proved.

\section{References}

1. J. M. ANDERSON and L. D. PITT, 'The boundary behaviour of Bloch functions and univalent functions', Michigan Math. J. 35 (1988) 313-320.

2. K. F. BARTH and P. J. RipPON, 'Angular limits of holomorphic functions of slow growth', $J$. London Math. Soc. (2) 45 (1992) 55-61.

3. K. F. BARTH and P. J. RIPPON, 'Minimal fine limits of subharmonic functions of slow growth', preprint, Open University, Milton Keynes, 1994.

4. K. F. BARTH, P. J. RIPPON, and L. SONS, 'Angular limits of holomorphic and meromorphic functions', J. London Math. Soc. (2) 42 (1990) 279-291.

5. R. BERMAN, 'Angular limits and infinite asymptotic values of analytic functions of slow growth', Illinois J. Math. 34 (1990) 845-858.

6. Y. S. CHOw, 'Convergence of sums of squares of martingale differences', Ann. of Math. Stat. 39 (1968) 123-133.

7. B. DAHLBERG, 'On the existence of radial boundary values for functions subharmonic in a Lipschitz domain', Indiana Univ. Math. J. 27 (1978) 515-526.

8. J. L. FERNÁNDEZ and J. G. LLORENTE, 'A note on the boundary behaviour of harmonic functions', J. London Math. Soc. (2) 46 (1992) 295-300.

9. B. Fuglede, Finely harmonic functions, Lecture Notes in Mathematics 289 (Springer, Berlin, 1972).

10. B. FUGLEDE, 'Asymptotic paths for subharmonic functions and polygonal connectedness of fine domains', Séminaire de Theorie du Potential, Paris, No. 5, Lecture Notes in Mathematics 814 (Springer, Berlin, 1980), pp. 97-116.

11. W. K. Hayman, Subharmonic functions, vol. 2 (Academic Press, London, 1990).

12. R. J. M. HoRnblower, 'A growth condition for the McLane class $\mathscr{A}$ ', Proc. London Math. Soc. (3) 23 (1971) 371-384.

13. R. J. M. Hornblower, 'Subharmonic analogues of McLane classes', Ann. Polon. Math. 26 (1972) $135-146$.

14. T. KILPELÄINEN and J. MALÝ, 'Supersolutions to degenerate elliptic equations on quasiopen sets', Comm. Partial Differential Equations 17 (1992) 371-405.

15. J. G. LlORENTE, 'Valores frontera de funciones armónicas. Geodésicas en superficies de Riemann', Tesis, Universidad Complutense de Madrid, 1993.

16. T. LYONS, 'Finely holomorphic functions', J. Funct. Anal. 37 (1980) 1-18.

17. N. G. MaKarov, 'Probability methods in conformal mapping', Algebra i Analiz. 1 (1989) 3-59.

18. G. R. MCLANE, 'Asymptotic values of holomorphic functions', Rice Univ. Studies 49 (1963).

19. NGUYEN-XUAN-LOC and T. WATANABE, 'Characterization of fine domains for a certain class of Markov processes with applications to Brelot harmonic spaces', Z. Wahrsch. Verw. Gebeite 21 (1972) 167-178.

20. A. N. ShIRYAyev, Probability (Springer, Berlin, 1984).

21. E. M. STEIN, Singular integrals and differentiability properties of functions (Princeton University Press, 1970).

22. W. F. STOUT, Almost sure convergence (Academic Press, London, 1974).

\section{J. L. Fernández and J. G. Llorente \\ Departamento de Matemáticas \\ Universidad Autónoma de Madrid 28049 Madrid \\ Spain}

J. Heinonen

Department of Mathematics

University of Michigan

Ann Arbor

Michigan 48109

U.S.A. 